\title{
Durable complete response rates following radiotherapy and immunotherapy combination in recurrent and metastatic head and neck squamous cell carcinoma: A retrospective single centre cohort study
}

\author{
Raj Khera ${ }^{1}$, Laura Feeney ${ }^{2}$, Martin Swinton ${ }^{1}$, Sam Rack ${ }^{1}$, Andrew Sykes ${ }^{1}$, and ROBERT \\ METCALF $^{1}$ \\ ${ }^{1}$ The Christie NHS Foundation Trust \\ ${ }^{2}$ Queen's University Belfast
}

February 24, 2022

\begin{abstract}
Key Points 1. This study reports clinical outcome data on 76 patients with platinum-resistant recurrent or metastatic head and neck squamous carcinoma treated with nivolumab, making it the largest published single centre case series of its kind. 2. Radiotherapy was administered alongside systemic therapy with nivolumab, defined as radiotherapy within 8 weeks of prior systemic therapy, in 16 of 76 patients (21\%). 3. Nivolumab was continued following radiotherapy completion due to ongoing clinical benefit from the drug in 9 of 16 patients (56\%). 4. Durable complete response following radio-immunotherapy was seen in 2 of the 9 patients $(22 \%)$ who continued nivolumab subsequent to radiotherapy. 5. Indications for radiotherapy included symptom control (3 of 9), oligoprogression (5 of 9) and incomplete response ( 1 of 9 ).
\end{abstract}

Durable complete response rates following radiotherapy and immunotherapy combination in recurrent and metastatic head and neck squamous cell carcinoma: A retrospective single centre cohort study

\section{Key Points}

1. This study reports clinical outcome data on 76 patients with platinum-resistant recurrent or metastatic head and neck squamous carcinoma treated with nivolumab, making it the largest published single centre case series of its kind.

2. Radiotherapy was administered alongside systemic therapy with nivolumab, defined as radiotherapy within 8 weeks of prior systemic therapy, in 16 of 76 patients $(21 \%)$.

3. Nivolumab was continued following radiotherapy completion due to ongoing clinical benefit from the drug in 9 of 16 patients (56\%).

4. Durable complete response following radio-immunotherapy was seen in 2 of the 9 patients (22\%) who continued nivolumab subsequent to radiotherapy.

5. Indications for radiotherapy included symptom control (3 of 9), oligoprogression (5 of 9) and incomplete response ( 1 of 9$)$.

\section{Key Words}

Head and neck cancer, nivolumab, immunotherapy, radiotherapy. 


\section{Introduction}

The advent of immune checkpoint inhibition has improved survival for patients with recurrent or metastatic platinum-resistant head and neck squamous cell carcinoma (HNSCC). In this setting, nivolumab has shown significantly improved outcome in comparison to chemotherapy (1). Evidence from pre-clinical studies suggests the addition of radiotherapy to immunotherapy may improve disease control (2). However, uncertainty remains as to whether combination treatment translates to improved outcomes in this clinical setting of recurrent or metastatic HNSCC. Currently, the decision to administer radiotherapy alongside systemic therapy in the palliative setting is made for selected patients on a case-by-case basis. However, there remains a need to define the additional clinical benefit of administration of radiotherapy alongside immunotherapy in this patient group.

\section{Objectives}

This study sought to determine the frequency with which radiotherapy was administered alongside nivolumab in patients with platinum-resistant recurrent or metastatic HNSCC and define the frequency of durable complete responses following radio-immunotherapy treatment.

\section{Method}

Design and Setting

This was a retrospective single-centre cohort study, conducted at a single tertiary cancer centre.

Participants and Outcome Measures

Clinical records of 76 patients with histologically confirmed HNSCC and confirmed disease progression within six month of prior platinum therapy were reviewed to identify patient who had received one or more doses of nivolumab in the recurrent or metastatic setting between February 2018 and August 2020. To determine the frequency with which multi-modality radio-immunotherapy was administered in this population, radiotherapy planning records were then reviewed to identify all patients who had received radiotherapy within 8 weeks of a prior dose of nivolumab. To determine the outcomes of patients following radio-immunotherapy, clinical data was reviewed in this subset of patients.

Ethical Considerations

The clinical record review of patients with platinum resistant HNSCC treated with nivolumab to identify those treated with radio-immunotherapy was conducted as part of the institutional clinical audit with the appropriate local institutional approvals. In accordance with the UK Policy Framework for Health and Social Care Research, subsequent data analysis was performed on anonymized data without any patient level identifiable information and no specific consent was acquired from each patient included in this study. For the inclusion of anonymized medical photography in the data presentation, individual patient consent was obtained under the MCRC Biobank Research Tissue Bank Ethics (NHS NW Research Ethics Committee $18 / \mathrm{NW} / 0092)$.

\section{Results}

As the primary aim of this study was to determine the outcomes for patients with HNSCC who received both nivolumab immunotherapy and radiotherapy, we first identified 76 patients at our institution who had received nivolumab immunotherapy with a diagnosis of platinum resistant recurrent or metastatic HNSCC between February 2018 and August 2020. 
On review of their radiotherapy planning records, we identified that among these 76 patients, 16 (21\%) had received radiotherapy within two months of a prior dose of nivolumab. The clinical characteristics of the patients who were treated with radiotherapy whilst in receipt of nivolumab are summarised in Table 1. These patients were predominantly male $(13 / 16,81 \%)$, and the median age at diagnosis was 61 (range 48-76 years). Metastatic disease at first presentation was recorded in 19\% (3/16), the remainder recurred following previous definitive treatment to localised disease. As primary treatment, $5(31 \%)$ patients had surgery, 5 (31\%) had chemoradiotherapy alone and $2(13 \%)$ had radiotherapy alone. Recurrent or metastatic disease developed within less than a year in 5/16 (31\%) patients range 2 to 41 months). 5 patients (31\%) were given nivolumab as second line systemic therapy following progression within 6 months of prior platinum as part of multi-modality treatment for localised disease. The remainder received nivolumab following progression on platinum based chemotherapy administered in the recurrent or metastatic setting.

The mean duration of nivolumab therapy prior to the initiation of radiotherapy was 16 weeks (range 0 to 43 weeks). On review of the systemic therapy records, of the 16 patients who received radiotherapy within two months of a prior dose of nivolumab, 9 (63\%) received further nivolumab subsequent to the radiotherapy and the remainder discontinued nivolumab at that point. The study schematic is shown in Figure 1.

The sites of disease which were irradiated included neck (7/16), abdomen (2), spine (3), and chest (1), skull base (1), oral cavity (1) left maxilla (1). The dose of radiation administered was 8 Gy for $7 / 16$ patients, 20 Gy 5/16, 27.5 Gy $1 / 16$ and 30 Gy for $3 / 16$ patients.

In those that continued with nivolumab subsequent to radiotherapy, two independent clinicians (A.S. and R.M) reviewed the case records of each patient to determine the clinical rationale for radiotherapy alongside nivolumab. For $3 / 9$ patients $(33 \%)$ radiotherapy was pre-planned alongside the start of nivolumab for symptom control. The indications for symptom control were haemostasis, metastatic cord compression management and pain control. For 5/9 patients (55\%), radiotherapy was administered for oligoprogression. For $1 / 9$ patients, radiotherapy was administered to a patient with a very good partial response to the only site of persistent disease.

Durable complete response following radio-immunotherapy was seen in 2 of the 9 patients who continued nivolumab subsequent to radiotherapy. For the first of these patients, previous treatment included concurrent chemo-radiotherapy with Carboplatin for T4N0M0 SCC of the maxilla. Nivolumab was being given for metastatic recurrence and although there was initial response, this patient received radiotherapy to a hilar lymph node which was the only site of progression, after 9 months of nivolumab, when there was radiological response elsewhere. There was a confirmed radiological response to radiotherapy (20 Gy in 5 fractions, Figure 2) and his patient subsequently continued with nivolumab achieving a complete response. At the time of analysis, this patient was alive at 29 months following initiation of nivolumab. The second patient who achieved a durable complete response was initially diagnosed with T3N2M0 SCC of the posterior oropharynx treated with chemoradiotherapy, with recurrence within 6 months of platinum chemoradiotherapy (Figure $3 \mathrm{~A}$ and $3 \mathrm{~B}$ ). There was a good partial response to nivolumab however, due to persistent clinically evident disease, radiotherapy (30 Gy in 10 fractions) was administered to the oro-mandibular residual disease after 22 weeks of nivolumab (Figure 3C and 3D). There was subsequent complete response clinically (Figure 3E and $3 \mathrm{~F}$ ) and radiologically (Figure $3 \mathrm{G}$ and $3 \mathrm{H}$ ) and the patient is alive at 26 months following start of nivolumab with no evidence of disease.

\section{Discussion}

Growing body of preclinical studies have demonstrated additional immunomodulatory effects with ionizing radiation which has promoted interest in potential synergy between radiotherapy and immunotherapy in the clinical setting (3). However, there remains an unmet clinical need to identify which patients with recurrent or metastatic HNSCC derive benefit from the combination of radiotherapy and immunotherapy.

In this study we have demonstrated that in a homogenous population of patients with platinum resistant 
recurrent and metastatic HNSCC treated with nivolumab, radiotherapy is administered in addition in $21 \%$ of patients, although radiotherapy and continued nivolumab was only administered in $12 \%$. We describe 2 of $9(22 \%)$ patients who received radio-immunotherapy then continued with nivolumab subsequently in whom durable complete response was seen. One of these patients had oligoprogression and the second had incomplete response so this observation would suggest that there is a signal of meaningful benefit for a subset of patients and provides the rationale for prospective studies to define the management of this patient group more clearly.

There are emerging data from prospective studies showing proof of concept that radiation may enhance immunotherapy response in other tumour types normally considered refractory to immune checkpoint blockade such as microsatellite stable colorectal and pancreatic adenocarcinoma (4). However, there are very limited prospective data evaluating radiotherapy alongside nivolumab in recurrent or metastatic HNSCC (5), In a randomized phase 2 trial (6), 62 patients with recurrent or metastatic HNSCC were treated with nivolumab with or without radiotherapy (SBRT) to a single metastatic site. They received 9 Gy $\times 3$ fractions between the first and second doses of nivolumab. This study did not demonstrate any difference in response rates with the addition of SBRT (34.5\% for nivolumab vs $29.0 \%$ for nivolumab with radiotherapy, $\mathrm{p}=\mathrm{ns}$ ), however other studies are ongoing in recurrent or metastatic HNSCC as well as the locally advanced setting (7).

There are limitations in this study. As all patients were treated at a single cancer centre, there is a high degree of confidence in data presented evaluating the frequency with which concomitant radiotherapy is administered and the clinical outcomes with this treatment. However, single-centre cohort studies will be biased by any variation in practice which would impact on patient selection for the combination of radioimmunotherapy. To further confirm these findings, other multi-centre studies would be valuable. Also, in the current study, the dose of radiotherapy varied between patients and was determined as per standard of care. However defining the optimal dose of radiotherapy and the context of previous irradiation may be critical in determining the immune response (8).

Conclusion

A sub-set of patients receiving radiotherapy-immunotherapy combination in platinum resistant HNSCC derived durable complete response associated with significant clinical benefit. This study highlights the need for a personalised approach in treatment decision making for patients who develop oligoprogression on nivolumab and provides a rationale for prospective clinical studies or registry studies to further address the role for multi-modality therapy in the recurrent or metastatic setting.

\section{References}

1. Ferris RL, Blumenschein G Jr, Fayette J, Guigay J, Colevas AD, Licitra L, et al., Nivolumab for Recurrent Squamous-Cell Carcinoma of the Head and Neck. N Engl J Med. 2016 Nov 10;375(19):18561867. doi: 10.1056/NEJMoa1602252. Epub 2016 Oct 8. PMID: 27718784; PMCID: PMC5564292.

2. Arina A, Gutiontov SI, Weichselbaum RR. Radiotherapy and Immunotherapy for Cancer: From "Systemic" to "Multisite". Clin Cancer Res. 2020 Jun 15;26(12):2777-2782. doi: 10.1158/1078-0432.CCR19-2034. Epub 2020 Feb 11. PMID: 32047000.

3. Daguenet E, Louati S, Wozny AS, Vial N, Gras M, Guy JB, et al., Radiation-induced bystander and abscopal effects: important lessons from preclinical models. Br J Cancer. 2020 Aug;123(3):339-348. doi: 10.1038/s41416-020-0942-3. Epub 2020 Jun 25. PMID: 32581341; PMCID: PMC7403362.

4. Parikh AR, Szabolcs A, Allen JN, Clark JW, Wo JY, Raabe M, et al., Radiation therapy enhances immunotherapy response in microsatellite stable colorectal and pancreatic adenocarcinoma in a phase II trial. Nat Cancer. 2021 Nov;2(11):1124-1135. doi: 10.1038/s43018-021-00269-7. Epub 2021 Nov 18. PMID: 35122060; PMCID: PMC8809884.

5. Qian JM, Schoenfeld JD. Radiotherapy and Immunotherapy for Head and Neck Cancer: Current Evidence and Challenges. Front Oncol. 2021 Feb 3;10:608772. doi: 10.3389/fonc.2020.608772. PMID: 
33614492; PMCID: PMC7886974.

6. McBride S, Sherman E, Tsai CJ, Baxi S, Aghalar J, Eng J, et al., Randomized Phase II Trial of Nivolumab With Stereotactic Body Radiotherapy Versus Nivolumab Alone in Metastatic Head and Neck Squamous Cell Carcinoma. J Clin Oncol. 2021 Jan 1;39(1):30-37. doi: 10.1200/JCO.20.00290. Epub 2020 Aug 21. PMID: 32822275; PMCID: PMC8462641.

7. Leidner R, Crittenden M, Young K, Xiao H, Wu Y, Couey MA, et al., Neoadjuvant immunoradiotherapy results in high rate of complete pathological response and clinical to pathological downstaging in locally advanced head and neck squamous cell carcinoma. J Immunother Cancer. 2021 May;9(5):e002485. doi: 10.1136/jitc-2021-002485. PMID: 33963014; PMCID: PMC8108690.

8. Botticelli A, Mezi S, Pomati G, Sciattella P, Cerbelli B, Roberto M, et al., The Impact of Locoregional Treatment on Response to Nivolumab in Advanced Platinum Refractory Head and Neck Cancer: The Need Trial. Vaccines (Basel). 2020 Apr 20;8(2):191. doi: 10.3390/vaccines8020191. PMID: 32326034; PMCID: PMC7349768.

\section{Hosted file}

Khera Figure 1.pptx available at https://authorea.com/users/462147/articles/557636durable-complete-response-rates-following-radiotherapy-and-immunotherapy-combination-inrecurrent-and-metastatic-head-and-neck-squamous-cell-carcinoma-a-retrospective-singlecentre-cohort-study

\section{Hosted file}

Khera Table 1.pptx available at https://authorea.com/users/462147/articles/557636-durablecomplete-response-rates-following-radiotherapy-and-immunotherapy-combination-inrecurrent-and-metastatic-head-and-neck-squamous-cell-carcinoma-a-retrospective-singlecentre-cohort-study

\section{Hosted file}

Khera Figure 2.pptx available at https://authorea.com/users/462147/articles/557636durable-complete-response-rates-following-radiotherapy-and-immunotherapy-combination-inrecurrent-and-metastatic-head-and-neck-squamous-cell-carcinoma-a-retrospective-singlecentre-cohort-study

\section{Hosted file}

Khera Figure 3.pptx available at https://authorea.com/users/462147/articles/557636durable-complete-response-rates-following-radiotherapy-and-immunotherapy-combination-inrecurrent-and-metastatic-head-and-neck-squamous-cell-carcinoma-a-retrospective-singlecentre-cohort-study 